Received: 3 May 2017

Accepted: 23 June 2017

Published online: 2 August 2017

\title{
Enhancing Modulation of Thermal Conduction in Vanadium Dioxide Thin Film by Nanostructured
}

\section{Nanogaps}

Hwan Sung Choe ${ }^{1}$, Joonki Suh ${ }^{1}$, Changhyun $\mathrm{Ko}^{1}$, Kaichen Dong ${ }^{1,2}$, Sangwook Lee ${ }^{1}$, Joonsuk Park $^{3}$, Yeonbae Lee ${ }^{1}$, Kevin Wang ${ }^{1}$ \& Junqiao Wu $\mathbb{B}^{1,4}$

Efficient thermal management at the nanoscale is important for reducing energy consumption and dissipation in electronic devices, lab-on-a-chip platforms and energy harvest/conversion systems. For many of these applications, it is much desired to have a solid-state structure that reversibly switches thermal conduction with high ON/OFF ratios and at high speed. Here we describe design and implementation of a novel, all-solid-state thermal switching device by nanostructured phase transformation, i.e., modulation of contact pressure and area between two poly-silicon surfaces activated by microstructural change of a vanadium dioxide $\left(\mathrm{VO}_{2}\right)$ thin film. Our solid-state devices demonstrate large and reversible alteration of cross-plane thermal conductance as a function of temperature, achieving a conductance ratio of at least 2.5. Our new approach using nanostructured phase transformation provides new opportunities for applications that require advanced temperature and heat regulations.

Control of heat conduction across interfaces would open up tremendous opportunities in advanced thermal energy management, to achieve super-stable temperature control, rapid temperature cycling, or pulsed thermal power operation $^{1-7}$. In this regard, thermal switches, which alter thermal conductance between ON and OFF states, have attracted considerable attention. In current technologies based on microfabrication, thermal switches are mainly achieved by connecting and breaking two surfaces with the motion of liquid droplets ${ }^{8-10}$ or solid membranes actuated with electric field ${ }^{11}$ or piezoelectric motors ${ }^{12}$. However, these non-monolithic approaches with moving parts often suffer from one or more of the following limitations: materials instability and toxicity, scalability, poor integrability with other devices, low ON/OFF ratio, low speed, and high energy consumption.

Solid-state phase transition materials such as vanadium dioxide $\left(\mathrm{VO}_{2}\right)^{13}, 14$, germanium antimony tellurium $(\mathrm{GeSbTe})$ ternary alloys ${ }^{15}$, and lead zirconium titanate $(\mathrm{PZT})^{16}$ are among the most attractive candidates for high-performance thermal switches: high speed of the phase transition ${ }^{17,18}$, intrinsic difference in thermal conductivities of each phase ${ }^{13,15,16}$, and reversible phase transitions activated by diverse external stimuli such as electric $^{16,19,20}$, optical ${ }^{21-23}$, mechanical ${ }^{24,25}$, or thermal ${ }^{13,15}$ ones. Demonstrated performance of the thermal switching based on these materials is, however, severely limited in one or more aspects of switching speed, ON/ OFF ratio, and/or energy efficiency. In this context, we develop a thermal switching structure (TSS) operated on the basis of open/close of a 1 2 nm nanogap activated by nanostructured phase transformation in a $\mathrm{VO}_{2}$ thin film. The approach is based on a recently developed, intriguing, multifunctional material composite that exhibits superior performance of micro-scale actuation. The system is composed of $\mathrm{VO}_{2}$, which possesses a ferroelastic phase transition, coupled mechanically to a structural material. The shape and mechanical, thermal, electrical and optical properties of the composite can be drastically and reversibly modified under external stimuli. At $68^{\circ} \mathrm{C}$, $\mathrm{VO}_{2}$ undergoes a coupled metal-insulator and rutile-monoclinic phase transition, where the effective lattice constant changes abruptly by up to $1 \sim 2 \%$ (Fig. 1b). Owing to the large work density of the $\mathrm{VO}_{2}$ phase transition, these

${ }^{1}$ Department of Materials Science and Engineering, University of California, Berkeley, Berkeley, CA, 94720, USA. ${ }^{2}$ Department of Precision Instrument, Tsinghua University, Beijing, 100084, China. ${ }^{3}$ Department of Materials Science and Engineering, Stanford University, Stanford, CA, 94305, USA. ${ }^{4}$ Materials Sciences Division, Lawrence Berkeley National Laboratory, Berkeley, CA, 94720, USA. Correspondence and requests for materials should be addressed to J.W. (email:wuj@berkeley.edu) 
a
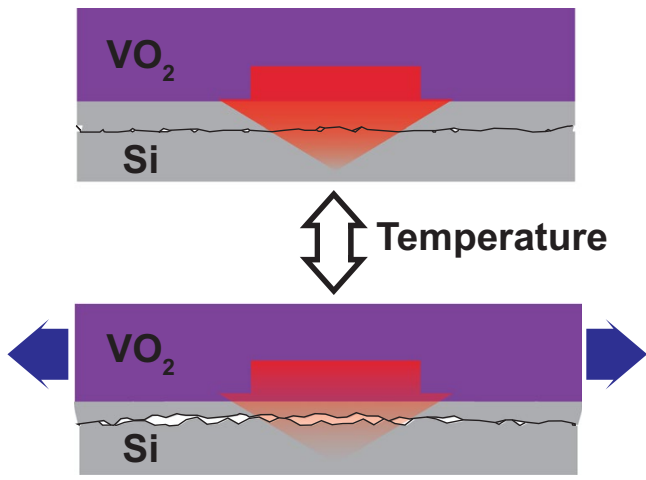

C

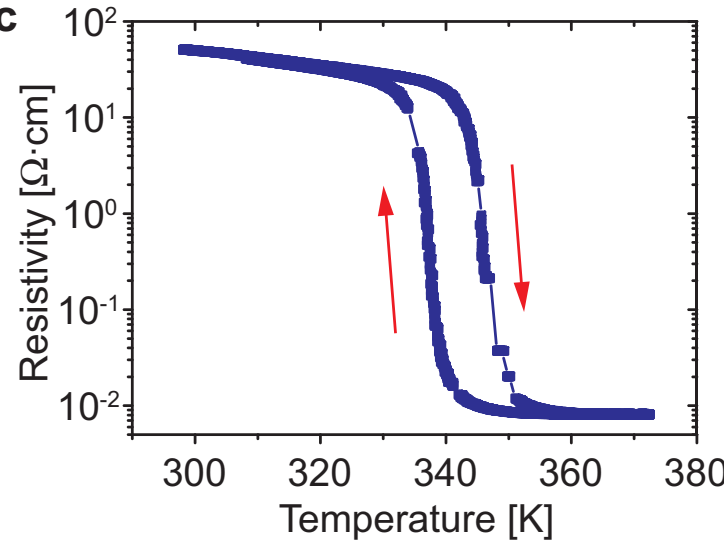

b

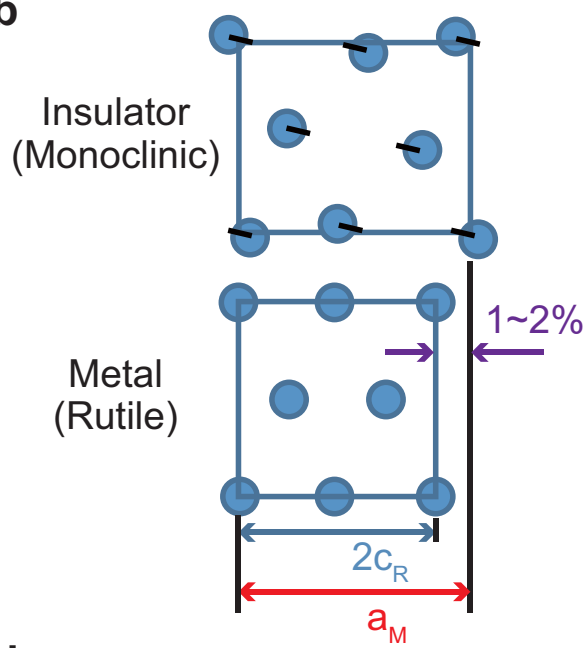

d

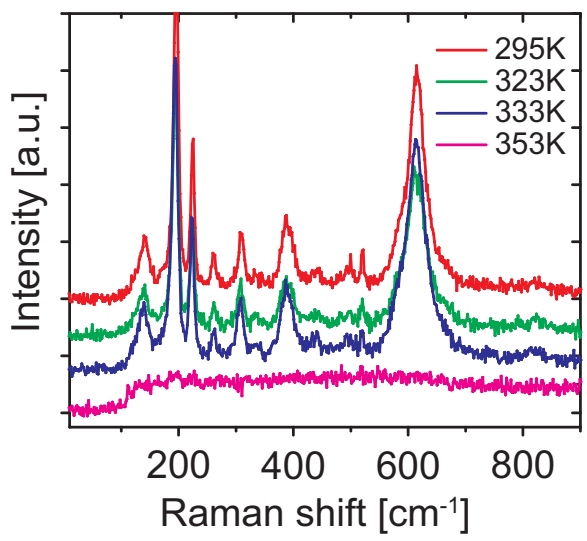

Figure 1. Concept of the nanostructured phase transformation and as-grown $\mathrm{VO}_{2}$ film properties. (a) Schematic of the nanostructured phase transformation with a nanogap, showing contact pressure and area modulated by the phase transition strain. (b) Illustration of the lattice structure change of $\mathrm{VO}_{2}$ across the phase transition between the insulating and metallic phases. In the schematics, the cyan circle indicates vanadium atom, and oxygen atoms are omitted for clarity. (c) Semi-log plot of resistivity of the $\mathrm{VO}_{2}$ film as a function of temperature during the heating and cooling process. (d) Raman spectra of the $\mathrm{VO}_{2}$ film at different temperatures showing the phase transition between 333 and $353 \mathrm{~K}$.

actuators can deliver simultaneously high force and large stroke of actuation, as opposed to other actuation mechanisms where only one is high. As shown in Fig. 1a, the TSS is mainly composed of a thin $\mathrm{VO}_{2}$ layer stacked onto a polycrystalline Si layer, with a nanogap created in the Si layer. The layers are deposited at temperatures higher than the $\mathrm{VO}_{2}$ phase transition temperature $\left(T_{P T}\right)$, such that at $T>T_{P T}$ the nanogap interface is largely conformal. As temperature is cooled across the phase transition to $T<T_{P T}$, the shape change of $\mathrm{VO}_{2}$ layer pulls the neighboring Si layer away from the Si layer below that, strongly reducing the contact pressure and area between these two layers. Heat conduction across the interface is thus proportionally reduced. Our TSS demonstrates dramatic enhancement of the thermal-switching ON/OFF ratio, by a factor of $\sim 6.7$, compared to $\mathrm{VO}_{2}$ film devices without incorporation of the nanogap.

\section{Results}

The $\mathrm{VO}_{2}$ thin films were grown using pulsed laser deposition on the surface of a poly-Si layer that was prepared with low-pressure chemical vapour deposition. Electrical characterization (Fig. 1c) of the $\mathrm{VO}_{2}$ film showed over three orders of magnitude change in its resistivity, and Raman spectroscopy (Fig. 1d) also confirmed the phase transition from the insulating phase to the metallic phase at around $341 \mathrm{~K}$, consistent with previous studies ${ }^{26}$.

In addition to the $\mathrm{VO}_{2}$ layer as the activation material, the other key component of the device is the nanogap embedded in the poly-Si layer that allows the open/close motion. This was created as illustrated schematically in Fig. 2a (see Experimental Section for details). A 20 nm sacrificial layer of low-temperature silicon dioxide (LTO) was sandwiched by the poly-Si layers, which was selectively removed using a hydrofluoric acid (HF) vapour etch. In particular, the HF vapour was utilized to etch the LTO entering from the side opening, causing the top poly-Si layer to collapse onto the bottom poly-Si layer with good conformation. Cross-sectional aberration-corrected high-resolution transmission electron microscopy (AC-HRTEM) of the interface area (Fig. 2b) revealed $1 \sim 2 \mathrm{~nm}$ interface roughness from both the top and bottom poly-Si layers, which indicates successful formation of a $1 \sim 2 \mathrm{~nm}$ nanogap in the structure. The existence of the designed nanogap in the device structure is further verified 
a

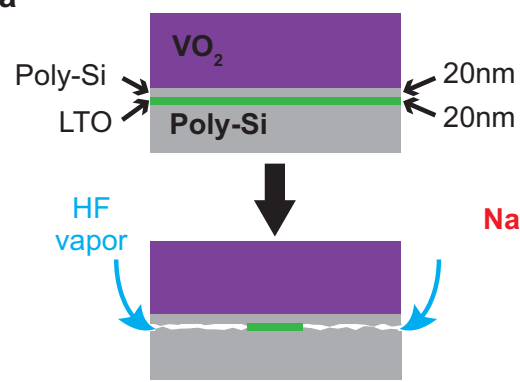

b

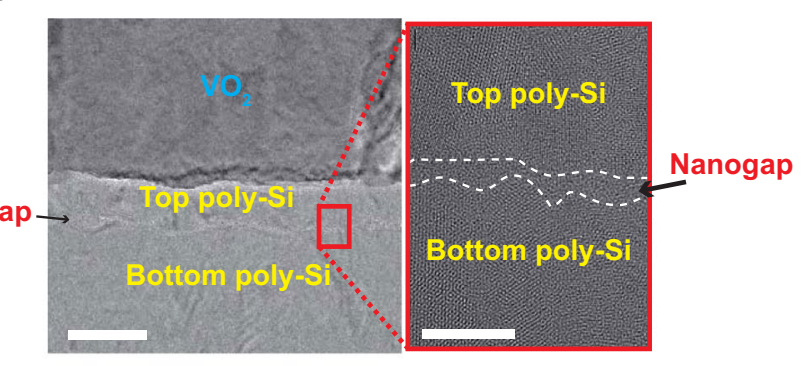

C

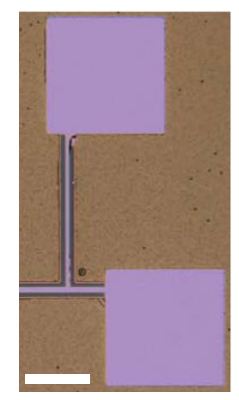

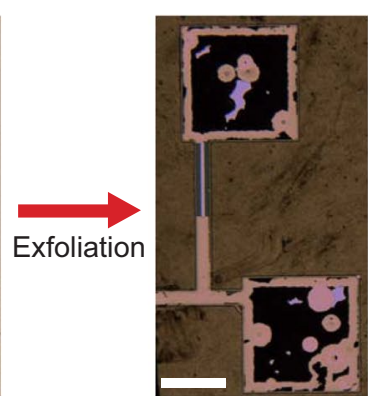

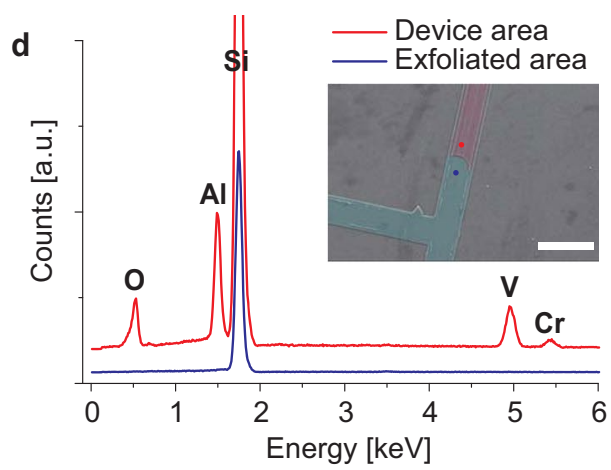

Figure 2. (a) Illustration of main process of the interface fabrication. The interface is formed by removal of the sacrificial LTO layer between the poly-Si layers with HF vapour etching. (b) Cross-sectional AC-HRTEM images of the interface area. The white dashed lines in the red box are drawn along the edge of the poly-Si layer. The continuum of atoms filling the nanogap were formed during the TEM sample preparation. Scale bar: $40 \mathrm{~nm}$ (left), and $8 \mathrm{~nm}$ (right). (c) Optical microscopic images of the electrodes of the thermal switch before (left) and after (right) tape exfoliation. Scale bar: $160 \mu \mathrm{m}$ (both left and right). (d) EDX spectra of exfoliated and un-exfoliated areas of the thermal switching device showing that the exfoliation indeed peels off the $\mathrm{VO}_{2}$ layer. Inset shows SEM image of the areas where EDX spectra were taken. False colors are added in exfoliated and unexfoliated areas for eye guidance. Scale bar: $80 \mu \mathrm{m}$.

as follows. First, the layer above the nanogap was able to be readily peeled off by mechanical exfoliation with a scotch tape. Figure 2c shows top-view optical images of the TSS before and after the mechanical exfoliation. Secondly, the exposed area, in contrast to the un-exfoliated area, shows absence of vanadium signal with energy dispersive $\mathrm{x}$-ray (EDX) analysis (Fig. 2d).

Modulation of the contact area across the nanogap of the TSS is expected to switch cross-plane thermal conduction of the structure, which was measured by the differential $3 \omega$ technique at variable background temperatures ${ }^{27}$. For clarity, the TSSs are hereby termed as " $\mathrm{VO}_{2}+\mathrm{NG"}$ " as they incorporate both the $\mathrm{VO}_{2}$ layer and the nanogap. To extract thermal conductance of the $\mathrm{VO}_{2}$-interface component within the multiple-layer stacked structure, we also prepared three control devices (Fig. S1): one with the same structure as the " $\mathrm{VO}_{2}+\mathrm{NG}$ " device but without the $\mathrm{VO}_{2}$ layer and the interface, hereby termed as "Si-only". In addition, an nanogap-only device ("NG") and $\mathrm{VO}_{2}$-only device (" $\mathrm{VO}_{2}$ ") were also prepared with otherwise identical structure under identical growth condition. According to the differential $3 \omega$ method, these different device structures in Fig. S1 can be utilized to investigate the modulation of thermal conduction across both the nanogap and the $\mathrm{VO}_{2}$ layer, driven by the neighboring structural phase transition, using the other structures as control devices.

As the width of the electric heater patterned for the $3 \omega$ technique (Fig. S9k) is much larger than the thickness of the entire stack, heat created in the top heater is considered to flow only vertically, resulting in a one-dimensional heat transfer problem. As a result, thermal response of a device containing any layer should be a linear combination of $\Delta T_{\text {reference }}$ and $\Delta T_{\text {layer }}$. For example, thermal resistance of the " $\mathrm{VO}_{2}+\mathrm{NG"} \mathrm{device} \mathrm{would} \mathrm{be} \mathrm{equal} \mathrm{to} \mathrm{the}$ "Si-only" device in series with the " $\mathrm{VO}_{2}+\mathrm{NG"} \mathrm{layer,} \mathrm{assuming} \mathrm{interfacial} \mathrm{resistances} \mathrm{negligible;}{ }^{27}$ consequently, effective thermal conductivity of the $\mathrm{VO}_{2}+\mathrm{NG}$ layer is given by

$$
\kappa_{V O_{2}+N G}=\frac{I_{\omega}^{2} \cdot R \cdot t_{V_{2}+N G}}{2 b l\left(\Delta T_{V_{2}+N G}-\Delta T_{S i-o n l y}\right)}
$$

where $t_{\mathrm{VO}_{2}+N G}, \Delta T_{S i-o n l y}, \Delta T_{\mathrm{VO}_{2}+N G}, 2 b$, and $l$ are the effective thickness of the $\mathrm{VO}_{2}$ and nanogap layer, tempera-

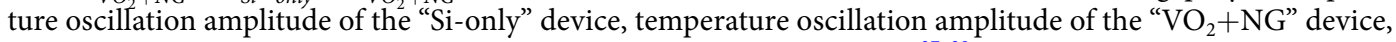
width, and length of the aluminum (Al) heater line (Fig. 9Sk), respectively ${ }^{27-29}$.

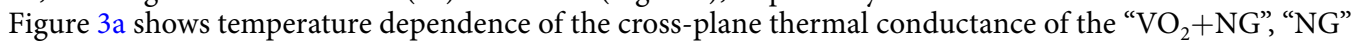
and " $\mathrm{VO}_{2}$ " layers measured with the differential $3 \omega$ method, using the "Si-only" device as the reference. For the 
a

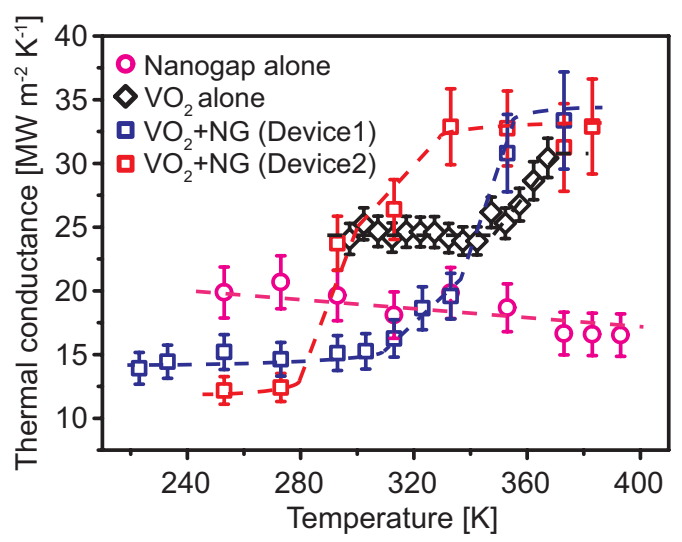

b

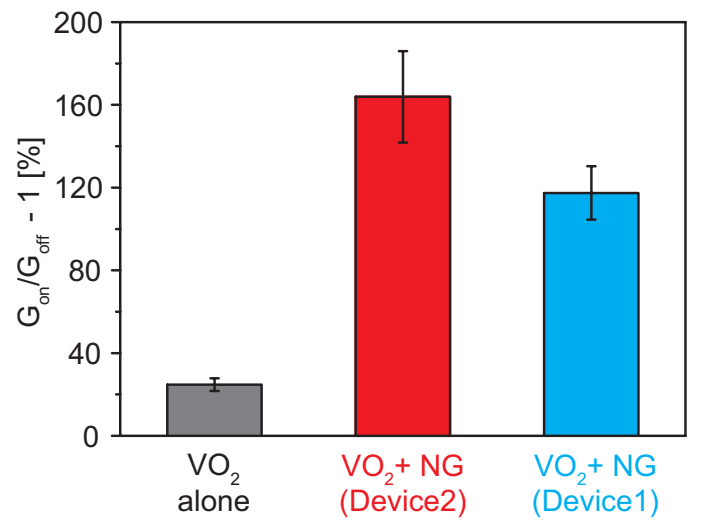

Figure 3. (a) Thermal conductance versus temperature plots of the nanogap, $\mathrm{VO}_{2}$ film alone, " $\mathrm{VO}_{2}+\mathrm{NG}$ " Device1, and " $\mathrm{VO}_{2}+\mathrm{NG"} \mathrm{Device2,} \mathrm{respectively.} 10 \%$ errors are estimated considering the error of thicknesses and measurements. The dashed lines are added as guides to the eye. (b) Comparison of thermal switching performance of $\mathrm{VO}_{2}$ film, "VO $2+\mathrm{NG"} \mathrm{Device2,} \mathrm{and} \mathrm{"} \mathrm{VO}_{2}+\mathrm{NG"} \mathrm{Device1} \mathrm{based} \mathrm{on} \mathrm{(a).}$

two " $\mathrm{VO}_{2}+\mathrm{NG}$ " devices, two different thicknesses of the topmost $\mathrm{Al}_{2} \mathrm{O}_{3}$ capping layer, being $25 \mathrm{~nm}$ (Device1) and $60 \mathrm{~nm}$ (Device2) respectively, were prepared as the electrical isolation layer between the $\mathrm{Al}$ heater and the underlying $\mathrm{VO}_{2}$ film. The phase transition temperature of the $\mathrm{VO}_{2}$ film in Device2 is lowered from the natural temperature $\left(68^{\circ} \mathrm{C}\right)$ to near room temperature as shown in Fig. S2. This is possibly attributed to the geometrical confinement effects by the upper $\mathrm{Al}_{2} \mathrm{O}_{3}$ capping layer and the lower poly-Si layer ${ }^{30}$. We analyze the behavior of each device as follows.

First of all, the $\mathrm{VO}_{2}$ layer shows $\sim 26 \%$ increase in thermal conductance as the $\mathrm{VO}_{2}$ switches from the insulating to the metallic phase, resulting in a $\mathrm{G}_{\text {on }} / \mathrm{G}_{\text {off }}$ of $\sim 1.26$ for the $\mathrm{VO}_{2}$ layer. From the thickness of the $\mathrm{VO}_{2}$ layer, the total thermal conductivity of the $\mathrm{VO}_{2}$ layer is estimated to increase by $\sim 0.76 \mathrm{~W} \mathrm{~m}^{-1} \mathrm{~K}^{-1}$ when going from the insulating to the metallic phase. Hall effect measurement reveals that the electrical conductivity of the metallic phase of the $\mathrm{VO}_{2}$ layer is $\sim 740 \Omega^{-1} \mathrm{~cm}^{-1}$. From the Wiedemann-Franz law with the Sommerfeld value of the Lorenz number, the electronic contribution to the thermal conductivity of the $\mathrm{VO}_{2}$ is estimated to be $\sim 0.67 \mathrm{~W} \mathrm{~m}^{-1} \mathrm{~K}^{-1}$ (Fig. S3), which is generally consistent with the increase in the total thermal conductance of the $\mathrm{VO}_{2}$ layer. This is in good agreement with previous work that shows the validity of the Wiedemann-Franz law in the metallic state of polycrystalline $\mathrm{VO}_{2}$ thin films ${ }^{13}$. Secondly, assuming that the effective average thickness of the nanogap is $\sim 2 \mathrm{~nm}$, supported by AC-HRTEM analysis (Fig. 2b), its thermal conductivity is determined from the "NG" device to be $\sim 0.02 \mathrm{~W} \mathrm{~m}^{-1} \mathrm{~K}^{-1}$, and is generally a constant as temperature varies, as shown in Fig. S3.

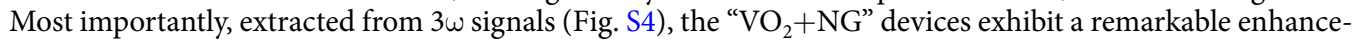
ment of the cross-plane thermal switching compared to the " $\mathrm{VO}_{2}$ " devices. The ON/OFF switching ratio of the thermal conductance $\left(\mathrm{G}_{\mathrm{on}} / \mathrm{G}_{\text {off }}\right)$ of the " $\mathrm{VO}_{2}$ ", " $\mathrm{VO}_{2}+\mathrm{NG}$ " Device1 and " $\mathrm{VO}_{2}+\mathrm{NG}$ " Device2 is 1.26, 2.39 and 2.75, respectively, corresponding to an enhancement of up to a factor of $\sim 6.7$ (26\% increase in thermal conductance in the " $\mathrm{VO}_{2}$ " device is improved to $175 \%$ in the " $\mathrm{VO}_{2}+\mathrm{NG"} \mathrm{Device)} \mathrm{when} \mathrm{the} \mathrm{nanogap} \mathrm{is} \mathrm{inserted} \mathrm{(Fig.} 3 \mathrm{~b}$ ). This result indicates that insertion of the nanogap into the poly-Si buffer layer significantly improves the thermal switching performance by utilizing the $\mathrm{VO}_{2}$ phase transition strain to modulate the contact area and, consequently, thermal conductance. Therefore, the mechanism of thermal conductance switching in the " $\mathrm{VO}_{2}+\mathrm{NG}$ " devices is different from that in the " $\mathrm{VO}_{2}$ " device, where the $\mathrm{ON}$-state is more thermally conductive than the OFF-state owing solely to free electrons released in the metallic phase.

The thermal switching of the " $\mathrm{VO}_{2}+\mathrm{NG"} \mathrm{nanomechanical} \mathrm{devices} \mathrm{is} \mathrm{reversible} \mathrm{and} \mathrm{stable.} \mathrm{Figure} \mathrm{4a} \mathrm{shows}$ thermal conductance of the Device1 during increase and decrease of background temperature. The conductance during the heating agrees well with that in the cooling with a small hysteresis. The hysteresis is narrower than that of the electrical conductivity in Fig. 1c, possibly due to the slow temperature variation in the former: the device was stabilized for 45 minutes prior to measurements of the thermal conductance at each temperature point. The nanostructuring and patterning for device fabrications may also introduce various defects in the $\mathrm{VO}_{2}$ layer, which can act as additional nucleation sites for the transition, effectively reducing the hysteresis. Plotted in Fig. $4 \mathrm{~b}$ is the repeated cycling of switching of the Device2, showing alteration of the thermal conductance between $12-14 \mathrm{MW} \mathrm{m}^{-2} \mathrm{~K}^{-1}$ for the insulating phase at $273 \mathrm{~K}$ and $26-28 \mathrm{MW} \mathrm{m}^{-2} \mathrm{~K}^{-1}$ for the metallic phase of $\mathrm{VO}_{2}$ at $373 \mathrm{~K}$. The device switches with good endurance and stability up to 100 cycles without any detectable degradation in performance.

Furthermore, to investigate the switching speed of our TSS, the phase transitions of the $\mathrm{VO}_{2}$ layer in a " $\mathrm{VO}_{2}+\mathrm{NG"} \mathrm{Device1} \mathrm{were} \mathrm{monitored} \mathrm{in} \mathrm{a} \mathrm{cryostat} \mathrm{by} \mathrm{a} \mathrm{Raman} \mathrm{spectroscope} \mathrm{with} \mathrm{temperature} \mathrm{control.} \mathrm{It} \mathrm{is} \mathrm{wor-}$ thy to note that phase transitions of the $\mathrm{VO}_{2}$ layer are responsible for the thermal switching of the " $\mathrm{VO}_{2}+\mathrm{NG}$ " Device1. Its Raman spectra as heating and cooling (Fig. S5) showed clear $\mathrm{VO}_{2}$ phase switching in $<\sim 10 \mathrm{~s}$ and $<\sim 30 \mathrm{~s}$ during $30 \mathrm{~K}$ heating and cooling, respectively, and demonstrated phase changes in $\sim 3 \mathrm{~s}$ during $10 \mathrm{~K}$ heating. The measured switching speed is significantly limited by, not an intrinsic device property, but ramp rates 
a

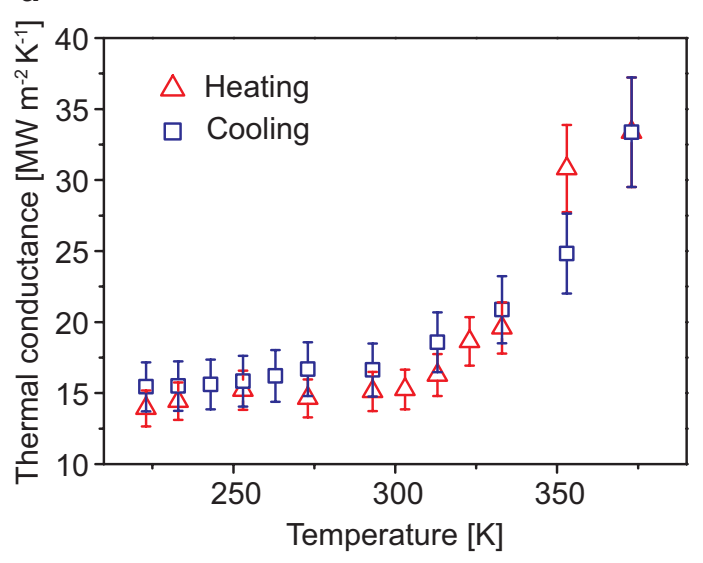

b

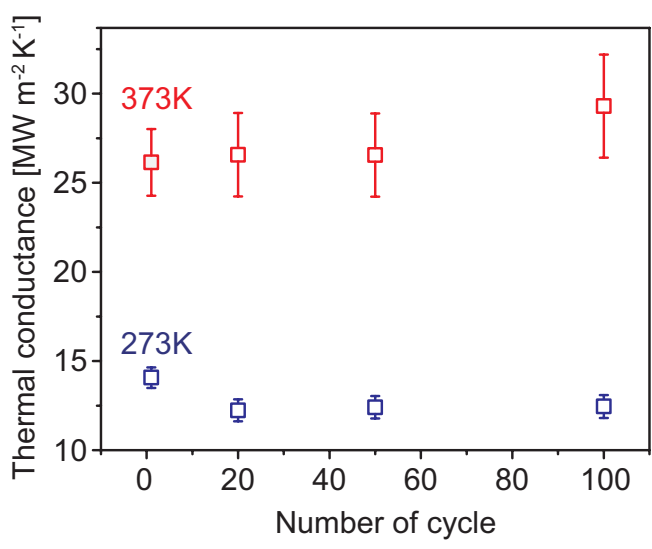

Figure 4. (a) Thermal conductance versus temperature plot of " $\mathrm{VO}_{2}+\mathrm{NG"} \mathrm{Device1} \mathrm{during} \mathrm{heating} \mathrm{and} \mathrm{cooling.}$

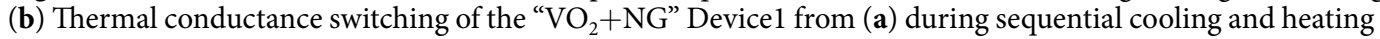
between $373 \mathrm{~K}$ and $273 \mathrm{~K}$, corresponding to alternation between the metal and insulator phases of $\mathrm{VO}_{2}$. The ramp rate of temperature was $5 \mathrm{~K} \mathrm{~min}^{-1}$ for both heating and cooling.

of background temperature during heating and cooling, as well as an acquisition time to obtain the Raman spectra. To evaluate the maximum thermal switching speed, we simulated $\mathrm{VO}_{2}$ phase switching in our TSS as applying a stepwise heat input by Joule heating on its top (see Methods for details). The results shown in Fig. S6 revealed that the maximum switching speed can be achieved up to $\sim 1 \mathrm{~ms}$. This switching speed is much slower than the intrinsic speed of both the metal-insulator transition (MIT) and the structural phase transition in $\mathrm{VO}_{2}{ }^{17,18}$, as the speed is limited by heat generation and dissipation in the structure. However, this speed is already comparable or faster than all reported solid thermal switches (see Table S1), and much faster than thermal switches involving liquids $^{8-10,12}$.

\section{Discussion}

The different thermal conduction of the " $\mathrm{VO}_{2}$ " and the " $\mathrm{VO}_{2}+\mathrm{NG}$ " devices implies that the inserted nanogap plays an active role in the thermal switching. To directly probe that, we performed in-situ SEM of the cross section of a " $\mathrm{VO}_{2}+\mathrm{NG"} \mathrm{device} \mathrm{under} \mathrm{temperature} \mathrm{variation} \mathrm{(Fig.} \mathrm{S7).} \mathrm{The} \mathrm{nanogap} \mathrm{is} \mathrm{not} \mathrm{directly} \mathrm{resolved} \mathrm{because} \mathrm{of}$ limitation in the SEM resolution. However, the thickness of the poly-Si layer above the nanogap varies reversibly with temperature cycling across the phase transition of $\mathrm{VO}_{2}$ (Fig. S7b,c). It is clear that the transformation strain of the $\mathrm{VO}_{2}$ film imposes stress onto the neighboring top poly-Si layer which is separated by the nanogap from the bottom poly-Si layer, hence modulating the nanogap size.

To further understand the thermal switching mechanism of the device, we simulated the heat conduction and radiation across the nanogap by analytical calculations. The total heat transfer coefficient attributed to radiative and conductive heat transfer across the nanogap between the poly-Si layers was first calculated using the Polder and Van Hove model $^{31,32}$. The calculation using the poly-Si permittivity ${ }^{33}$ show radiative heat transfer coefficient carried by far-field waves or evanescent waves (Fig. S8a,b). Not surprisingly, the results at both temperatures (where the $\mathrm{VO}_{2}$ is in two different phases) reveal that radiative heat transfer grows exponentially as the nanogap is reduced from 10 to below $0.1 \mathrm{~nm}$. Due to $1 \sim 2 \mathrm{~nm}$ roughness of the poly-Si surface (Fig. $2 \mathrm{~b}$ ) and hence the non-uniform thickness of the nanogap at nanoscale, we calculated the total heat transfer coefficient across a simplified gap structure, based on the results in Fig. S8a,b: a Si pyramid approaching a plane of Si (inset of Fig. S8c). We assume an initial gap size of $2 \mathrm{~nm}$, and that the heat conduction under direct contact is equal to the amount of radiative heat transfer at $1 \AA$ gap $^{34}$. Figure S8c,d show the calculated heat transfer coefficients of radiation and conduction across the structure as the gap is closed when the pyramid approaches the other plane. The result shows that the total heat transfer is by radiation at $2 \mathrm{~nm}$ gap at $253 \mathrm{~K}$ (OFF state of the thermal switch), and the conduction component of heat transfer increases as the gap is reduced. The experimental result of $\mathrm{G}_{\text {on }} / \mathrm{G}_{\text {off }} \sim$ 2.75 from our TSS (red block in Fig. 3b) can be reproduced if the nanogap shrinks from $2 \mathrm{~nm}$ in the OFF state to $\sim 1.5 \mathrm{~nm}$ in the $\mathrm{ON}$ state. We note that, as both the direct heat conduction and near-field heat radiation contribute to the total heat transfer, the thermal conduction would not be zero even when the nanogap is completely open. This limits the maximum $G_{\text {on }} / G_{\text {off }}$ from divergence. In the ideal scenario assuming that the nanogap can vary from $\sim 1 \mu \mathrm{m}$ (fully open) to a fully conform contact, the calculation shows that $G_{\text {on }} / G_{\text {off }}$ reaches up to two orders of magnitude. Overall, both the in-situ SEM imaging and the simulation support the active role of the nanogap in the heat transfer modulation.

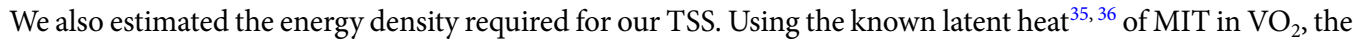
consumed energy density is $\sim 47 \mathrm{pJ} \mu^{-2}$ for a $200 \mathrm{~nm} \mathrm{VO}_{2}$ layer used. Assuming the same active layer thickness ( $200 \mathrm{~nm}$ for all devices), this value is about $1 / 5$ of that in thermal switches based on the GeSbTe phase change materials system ${ }^{15}$, but a factor of 40 higher than that based on the PZT ferroelectric system ${ }^{16}$. Compared to the PZT devices, the merit of our device therefore lies in its higher ON/OFF ratio, as shown in Table S1, with sacrifice in higher energy consumption. 
In summary, we demonstrated a thermal switching in a solid-state nanomechanical thin-film structure based on nanostructured phase transformation. A high ON/OFF ratio of $\sim 2.75$ in cross-plane thermal conductance is achieved in the device as the contact area across a nanogap is modulated by the phase transition strain of the $\mathrm{VO}_{2}$ layer. This is a $670 \%$ enhancement from the thermal switching based on the metal-insulator transition of $\mathrm{VO}_{2}$ alone. $\mathrm{VO}_{2}$ is non-toxic, and thermally and chemically stable $\left(\mathrm{VO}_{2}\right.$ micro-actuators show no degradation after millions of operation) ${ }^{37}$, its phase transition in a TSS is with high speed (intrinsically $\sim$ picosec, leading to device speed $\sim 1 \mathrm{kHz}$ limited purely by thermal dissipation $)^{38}$, and the transition temperature can be shifted to other temperatures with chemical doping ${ }^{19}$. The fact that the phase transition can be driven thermally ${ }^{13}$, optically ${ }^{21}$, electrically ${ }^{19}$ and electrostatically ${ }^{20}$ promises versatility and flexibility in operation and performance of the thermal switch in nanoelectromechanical systems. The demonstrated solid-state thermal switching is expected to open new opportunities for applications that require advanced temperature and heat regulations.

\section{Methods}

$\mathrm{VO}_{2}$ thin films growth and characterization. All $\mathrm{VO}_{2}$ thin films used in this study were grown on either lightly p-doped $(20-30 \Omega \cdot \mathrm{cm})$ single crystalline (100) silicon substrate or undoped polycrystalline silicon thin films by pulsed laser deposition (PLD). A Krypton fluoride excimer laser (248 nm wavelength) was focused on a $\mathrm{VO}_{2}$ target (pressed $99.9 \%$ pure, powder) with a pulse repetition rate of $5 \mathrm{~Hz}$ and a fluence of $350 \mathrm{~mJ} \mathrm{~cm} \mathrm{c}^{-2}$. The deposition was done at $500^{\circ} \mathrm{C}$ in $10 \mathrm{mTorr}$ with $2 \mathrm{sccm}$ oxygen gas flow. After finishing the deposition, the sample was cooled down to room temperature at a rate of $10^{\circ} \mathrm{C} \mathrm{min}^{-1}$ with retaining oxygen gas pressure. The thickness, crystal orientation, resistance, and phase of the as-grown film were characterized by SEM, XRD, electrical transport measurement, and Raman spectroscopy, respectively. For the resistance measurement, two probes made contacts at the opposite sides of the film grown on undoped poly-Si film, and the film resistance was recorded at variable substrate temperature. The results are used for the resistivity calculation of the $\mathrm{VO}_{2}$ film (Fig. 1c) with the information of its dimension.

Device fabrication and AC-HRTEM characterization. The high-quality, undoped poly-Si and low temperature undoped silicon oxide (LTO) were deposited by low-pressure chemical vapour deposition at $615^{\circ} \mathrm{C}$ and $450^{\circ} \mathrm{C}$, respectively, using reactive silane and oxygen gases in $300 \mathrm{mTorr}$ (Fig. S9a). The 120 or $200 \mathrm{~nm} \mathrm{VO}$ film was deposited by PLD on underlying $120 \mathrm{~nm}$ poly-Si $/ 20 \mathrm{~nm} \mathrm{LTO} / 20 \mathrm{~nm}$ poly-Si thin-film stack (Fig. S9b), and then $70 \mathrm{~nm} \mathrm{Al}_{2} \mathrm{O}_{3}$ using atomic layer deposition (ALD) (vacuum, $200^{\circ} \mathrm{C}$, trimethylaluminum and DI water as precursors) and $\mathrm{Cr}(1 \mathrm{~nm}) / \mathrm{Au}(70 \mathrm{~nm})$ layer by electron-beam evaporation to protect underlying layers during further etching processes. (Fig. S9c). The interface forms from HF vapour etch (uEtch, SPTS Technologies) (Fig. S9e), following wet chemical etch with BHF and selective RIE ( $100 \mathrm{~W}, 13 \mathrm{sccm} \mathrm{SF}$ and $21 \mathrm{sccm} \mathrm{He}$ ) to remove the $\mathrm{Al}_{2} \mathrm{O}_{3}$ and poly-Si/VO layers, respectively (Fig. S9d), to allow the etchant to access the LTO layer. The Cr/Au protection layers were etched out using CR-7 chromium and TFA gold etchant, respectively (Fig. S9f). Right after the etching process, $\mathrm{Si}_{3} \mathrm{~N}_{4}(10 \mathrm{~nm})$ and $\mathrm{Al}_{2} \mathrm{O}_{3}(60$ or $25 \mathrm{~nm})$ were deposited by plasma-enhanced chemical vapour deposition (20 W, 0.9 Torr, $200^{\circ} \mathrm{C}, 30 \mathrm{sccm} \mathrm{NH}$ and $100 \mathrm{sccm} 10 \% \mathrm{SiH}_{4}$ in Ar) and ALD, respectively (Fig. S9g). In particular, the thin $\mathrm{Si}_{3} \mathrm{~N}_{4}$ layer was for prevention of the $\mathrm{Al}_{2} \mathrm{O}_{3}$ deposition into the localized nanogaps at the interface during post-ALD process, and the additional $\mathrm{Al}_{2} \mathrm{O}_{3}$ layer was for electrical isolation between the $\mathrm{VO}_{2}$ film and the electrodes. Finally, the electrode pattern of $\mathrm{Cr}(1 \mathrm{~nm}) / \mathrm{Al}(100 \mathrm{~nm})$ for $3 \omega$ method was metallized by standard photolithography and electron beam evaporation (Fig. S9h). The yield of the TSS demonstrating proper thermal switching operation was $75 \%$ ( 3 out of 4 devices).

The samples for cross-sectional AC-HRTEM (Titan, FEI) analysis were prepared by focused ion beam (Helio NanoLab, FEI) and lift-out technique. All HRTEM images (Fig. 2b and Fig. S9j) were taken at $300 \mathrm{kV}$ with spherical aberration correction. For high contrast of the interface, $>40 \mathrm{~nm}$ defocus and an objective aperture were used.

Sample preparation and experimental setup for the $\mathbf{3} \omega$ method. All devices were mounted on a 24-pin chip carrier with a class-A platinum thin-film resistance temperature detector (RTD) by conductive silver paint. It is worthy to note that the RTD was employed to accurately read the temperature right on the devices. $\mathrm{Al}$ wire bonding and silver conductive epoxy $\left(\mathrm{EPO}-\mathrm{TEK}^{\circledR} \mathrm{H} 20 \mathrm{E}\right)$ were used for stable electrical connections between the chip carrier and the $\mathrm{Al}$ heater over a wide range of temperatures. The chip carrier with devices was loaded into a cryostat (CCS-400H/204, Janis) which was connected to a vacuum turbopump (T-Station 75 , Edwards), a temperature controller (Model 331, Lakeshore) and a home-made BNC box. For $3 \omega$ voltage measurement of the devices, the cryostat was evacuated to $\sim 1 \times 10^{-7}$ Torr, and a lock-in amplifier (SR830, Stanford Research Systems), V to I converters, a multiplying DAC, and a high precision resistors $(100 \Omega, \pm 0.005 \%$ resistance tolerance, $\pm 0.05 \mathrm{ppm}^{\circ} \mathrm{C}^{-1}$ temperature coefficient) were wired as shown in Fig. S10. We have used a commercial fused silica substrate to benchmark our $3 \omega$-system, and found a thermal conductivity of $1.22 \mathrm{~W} \mathrm{~m}^{-1} \mathrm{~K}^{-1}$ (Fig. S11) very close to the known value. Before starting to record data, the global temperature was raised up to $423 \mathrm{~K}$ and stayed there for $\sim 30 \mathrm{~min}$ to anneal the devices. For the temperature coefficient of resistance (TCR) of each $\mathrm{Al}$ heater at the target temperature $\mathrm{T}_{0}, \mathrm{I}-\mathrm{V}$ curves were collected at five different temperatures, $\mathrm{T}_{0}-4, \mathrm{~T}_{0}-2$, $\mathrm{T}_{0}, \mathrm{~T}_{0}+2$, and $\mathrm{T}_{0}+4 \mathrm{~K}$ after temperature stabilization for 30 minutes at each temperature. The resistances of the heater at the five temperatures were obtained by a linear fitting to the I-V curve. Consequently, The TCR at $\mathrm{T}_{0}$ was determined by a linear-fitting. The $3 \omega$ data acquisition at $\mathrm{T}_{0}$ was carried out after temperature stabilization for 45 minutes, too. It is worth noting that the nanogap modulation occurs prior to the $3 w$ measurements, such that during the measurements, the contact area is not actuating, and the measurements in Figs 3 and 4 are static/ equilibrium instead of dynamic.

Experimental setup and condition of the Raman spectroscopic characterization for dynamic switching measurement. All devices characterized by Raman spectroscopy were loaded into a cryostat 
(THMS600, Linkam Scientific Instruments). The temperature in the cryostat was precisely controlled by an embedded heater in the system and liquid nitrogen from outsourcing for heating and cooling, respectively. The measurements of $\mathrm{VO}_{2}$ phases in a " $\mathrm{VO}_{2}+\mathrm{NG}$ " device, demonstrated in Fig. S5, were performed using a $50 \times$ objective lens on a Renishaw micro-Raman/PL system equipped with an excitation laser $(\lambda \sim 488 \mathrm{~nm})$ which was focused on the area right next to the $3 \omega$ electrode (Fig. S2a). 190- $\mu \mathrm{W}$ laser power allowed acquiring Raman signals from the " $\mathrm{VO}_{2}+\mathrm{NG}$ " device within $0.5 \mathrm{~s}$ without any damage. The device was heated and cooled with maximum ramp rate limited by the cryostat system.

Simulation of dynamic thermal switching of a TSS. Considering our " $\mathrm{VO}_{2}+\mathrm{NG}$ " device, we performed two dimensional time-dependent numerical calculation with a thin film structure of $100 \mathrm{~nm} \mathrm{Al} \mathrm{heating}$ layer, $95 \mathrm{~nm} \mathrm{Al} \mathrm{O}_{3}$ layer, $200 \mathrm{~nm} \mathrm{VO}$ actuation layer and $20 \mathrm{~nm}$ poly-Si layer, as described in the inset of Fig. S6. Its total length was $500 \mu \mathrm{m}$ with temperature constant anchor regions at both ends (anchor length $=20 \mu \mathrm{m}$ ). Set on $273 \mathrm{~K}$ for an initial temperature of the environment and the whole structure, and the anchors used a fixed temperature of $273 \mathrm{~K}$ as the boundary condition. The temperature-dependent heat flux of the nanogap interface is derived from the calculated results (Fig. S8), while the heat fluxes of the other boundaries are $5 \mathrm{Wm}^{-2} \mathrm{~K}^{-1}$ ) to imitate the air cooling in the ambient environment. The parameters for $\mathrm{VO}_{2}$ properties such as density ${ }^{39}$, heat capacity (including latent heat) ${ }^{40}$, Young's modulus and Poisson's ratio ${ }^{41}$, electrical conductivity and thermal conductivity are carefully chosen using literature and experimental or analytical values in this work. The material properties of $\mathrm{Al}$, poly-Si and $\mathrm{Al}_{2} \mathrm{O}_{3}$ are based on build-in parameters in the COMSOL Multiphysics. Their thermal expansion is neglected, because thermal expansion is much weaker in the experiments compared to the transformaiton strain during the $\mathrm{VO}_{2}$ phase transition ${ }^{37}$. A stepwise voltage input was supplied to the $\mathrm{Al}$ layer, which heats up the entire system to $\sim 343 \mathrm{~K}$. It should be noted that the temperature of $\mathrm{VO}_{2}$ is evaluated at the geometrical central point of the $\mathrm{VO}_{2}$ layer.

Data Availability. The datasets generated during and/or analysed during the current study are available from the corresponding author upon request.

\section{References}

1. Vassighi, A. \& Sachdev, M. Thermal and Power Management of Integrated Circuits. (Springer, 2006).

2. Dames, C. Thermal materials: Pulling together to control heat flow. Nat Nanotechnol. 7, 82-83, doi:10.1038/nnano.2012.4 (2012).

3. Kim, H.-S., Liao, H.-H., Song, H. O. \& Kenny, T. W. Variable thermal resistors (VTR) for thermal management of chip scale atomic clocks (CSAC). IEEE Int. Conf. MEMS, 4, doi:10.1109/MEMSYS.2008.4443790 (2008).

4. Li, N. et al. Colloquium: Phononics: Manipulating heat flow with electronic analogs and beyond. Rev. Mod. Phys. 84, 1045-1066, doi:10.1103/RevModPhys.84.1045 (2012).

5. Cottrill, A. L. \& Strano, M. S. Analysis of Thermal Diodes Enabled by Junctions of Phase Change Materials. Adv. Energy Mater. 5, 1500921, doi:10.1002/aenm.201500921 (2015).

6. Fangyuan Sun et al. Molecular Bridge Enables Anomalous Enhancement in Thermal Transport across Hard-Soft Material Interfaces. Adv. Mater. 26, 6093, doi:10.1002/adma.201400954 (2014).

7. Guoqing Xin et al. Large-Area Freestanding Graphene Paper for Superior Thermal Management. Adv. Mater. 26, 4521, doi:10.1002/ adma.201400951 (2014).

8. McLanahan, A. R., Richards, C. D. \& Richards, R. F. A dielectric liquid contact thermal switch with electrowetting actuation. J. of Micromech. Microeng. 21, 104009, doi:10.1088/0960-1317/21/10/104009 (2011).

9. Tsukamoto, T., Esashi, M. \& Tanaka, S. Long working range mercury droplet actuation. J. Micromech. Microeng. 19, 094016, doi:10.1088/0960-1317/19/9/094016 (2009).

10. Cho, J., Wiser, T., Richards, C., Bahr, D. \& Richards, R. Fabrication and characterization of a thermal switch. Sens. Actuat. A-Phys. 133, 55-63, doi:10.1016/j.sna.2006.03.033 (2007).

11. Slater, T., Van Gerwen, P., Masure, R., Preud'homme, F. \& Baert, K. Thermomechanical characteristics of a thermal switch. Sens. Actuat. A-Phys. 53, 423, doi:10.1016/0924-4247(96)80165-8 (1996).

12. Cho, J., Richards, C., Bahr, D., Jiao, J. \& Richards, R. Evaluation of contacts for a MEMS thermal switch. J. Micromech. Microeng. 18, 105012, doi:10.1088/0960-317/18/10/105012 (2008).

13. Oh, D.-W., Ko, C., Ramanathan, S. \& Cahill, D. G. Thermal conductivity and dynamic heat capacity across the metal-insulator transition in thin film $\mathrm{VO}_{2}$. Appl. Phys. Lett. 96, 151906, doi:10.1063/1.3394016 (2010).

14. Savo, S. et al. Reconfigurable anisotropy and functional transformations with $\mathrm{VO}_{2}$-based metamaterial electric circuits. Phys. Rev. $B$ 91, 134105, doi:10.1103/PhysRevB.91.134105 (2015).

15. Shin, S., Kim, H. K., Song, J., Choi, D. J. \& Cho, H. H. Phase-dependent thermal conductivity of $\mathrm{Ge}_{1} \mathrm{Sb}_{4} \mathrm{Te}_{7}$ and $\mathrm{N}_{\mathrm{G}} \mathrm{Ge}_{1} \mathrm{Sb}_{4} \mathrm{Te}_{7}$ for phase change memory applications. J. Appl. Phys 107, 033518, doi:10.1063/1.3294694 (2010).

16. Ihlefeld, J. F. et al. Room-temperature voltage tunable phonon thermal conductivity via reconfigurable interfaces in ferroelectric thin films. Nano Lett. 15, 1791-1795, doi:10.1021/nl504505t (2015).

17. Pashkin, A. et al. Ultrafast insulator-metal phase transition in $\mathrm{VO}_{2}$ studied by multiterahertz spectroscopy. Phys. Rev. B 83, doi:10.1103/PhysRevB.83.195120 (2011).

18. Baum, P., Yang, D. S. \& Zewail, A. H. 4D visualization of transitional structures in phase transformations by electron diffraction. Science 318, 788-792, doi:10.1126/science.1147724 (2007).

19. Gu, Q., Falk, A., Wu, J., Ouyang, L. \& Park, H. Current-driven phase oscillation and domain wall propagation in $\mathrm{W}_{\mathrm{x}} \mathrm{V}_{1-\mathrm{x}} \mathrm{O}_{2}$ nanobeams. Nano Lett. 7, 363-366, doi:10.1021/nl0624768 (2007).

20. Nakano, M. et al. Collective bulk carrier delocalization driven by electrostatic surface charge accumulation. Nature 487, 459, doi:10.1038/nature11296 (2012).

21. Rini, M. et al. Photoinduced phase transition in $\mathrm{VO}_{2}$ nanocrystals: ultrafast control of surface-plasmon resonance. Opt. Lett. 30, 558-560, doi:10.1364/OL.30.000558 (2005).

22. Lourembam, J. et al. Evidence for Photoinduced Insulator-to-Metal transition in B-phase vanadium dioxide. Sci. Rep. 6, doi:10.1038/ srep25538 (2016).

23. Tao, Z. S. et al. The nature of photoinduced phase transition and metastable states in vanadium dioxide. Sci. Rep. 6, doi:10.1038/ srep38514 (2016)

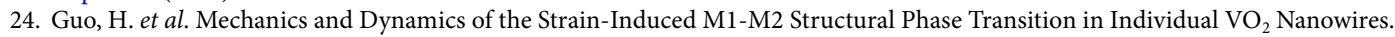
Nano Lett. 11, 3207-3213, doi:10.1021/nl201460v (2011).

25. Kim, M. W. et al. Substrate-mediated strain effect on the role of thermal heating and electric field on metal-insulator transition in vanadium dioxide nanobeams. Sci. Rep. 5, doi:10.1038/srep10861 (2015). 
26. Pan, M. et al. Raman study of the phase transition in $\mathrm{VO}_{2}$ thin films. J. Cryst. Growth 268, 178-183, doi:10.1016/j. jcrysgro.2004.05.005 (2004).

27. Dames, C. Measuring the thermal conductivity of thin films: 3 omega and related electrothermal methods. Annual Review of Heat Transfer 16, 7-49, doi:10.1615/AnnualRevHeatTransfer.v16.20 (2013).

28. Cahill, D. G., Katiyar, M. \& Abelson, J. R. Thermal conductivity of a-Si:H thin films. Phys. Rev. B 50, 6077-6081, doi:10.1103/ PhysRevB.50.6077 (1994).

29. Lee, J., Lim, J. \& Yang, P. Ballistic phonon transport in holey silicon. Nano Lett. 15, 3273-3279, doi:10.1021/acs.nanolett.5b00495 (2015).

30. Viswanath, B., Ko, C., Yang, Z. \& Ramanathan, S. Geometric confinement effects on the metal-insulator transition temperature and stress relaxation in $\mathrm{VO}_{2}$ thin films grown on silicon. J. Appl. Phys. 109, 063512, doi:10.1063/1.3556756 (2011).

31. Polder, D. \& Van Hove, M. Theory of Radiative Heat Transfer between Closely Spaced Bodies. Phys. Rev. B 4, 3303-3314, doi:10.1103/PhysRevB.4.3303 (1971).

32. Ottens, R. S. et al. Near-Field Radiative Heat Transfer between Macroscopic Planar Surfaces. Phys. Rev. Lett. 107, doi:10.1103/ PhysRevLett.107.014301 (2011).

33. Ho, J. H., Lee, C. L., Lei, T. F. \& Chao, T. S. Ellipsometry measurement of the complex refractive index and thickness of polysilicon thin films. J. Opt. Soc. Am. A 7, 196-205, doi:10.1364/JOSAA.7.000196 (1990).

34. Chiloyan, V., Garg, J., Esfarjani, K. \& Chen, G. Transition from near-field thermal radiation to phonon heat conduction at subnanometre gaps. Nat. Commun. 6, 6755, doi:10.1038/ncomms7755 (2015).

35. Berglund, C. N. \& Guggenheim, H. J. Electronic properties of $\mathrm{VO}_{2}$ near the semiconductor-metal transition. Phys. Rev. 185, 1022-1033, doi:10.1103/PhysRev.185.1022 (1969).

36. Cao, J. et al. Strain engineering and one-dimensional organization of metal-insulator domains in single-crystal vanadium dioxide beams. Nat. Nanotechnol. 4, 732-737, doi:10.1038/NNANO.2009.266 (2009).

37. Liu, K. et al. Giant-Amplitude, High-Work Density Microactuators with Phase Transition Activated Nanolayer Bimorphs. Nano Lett. 12, 6302, doi:10.1021/nl303405g (2012).

38. Liu, K. et al. Powerful, Multifunctional Torsional Micro Muscles Activated by Phase Transition. Adv. Mater. 26, 1746-1750, doi:10.1002/adma.201304064 (2014).

39. Stefanovich, G., Pergament, A. \& Stefanovich, D. Electrical switching and Mott transition in VO2. J. phys-Condens. Mat. 12, 8837-8845 0.1088/0953-8984/12/41/310. (2000).

40. Zhong, X. Semiconductor-metal Transition in Epitaxial $\mathrm{VO}_{2}$ Thin Films on $\mathrm{TiO}_{2}(100)$ Considering a Phase Equilibrium. (University of Alabama Libraries, 2013).

41. Cabrera, R., Merced, E. \& Sepulveda, N. Performance of Electro-Thermally Driven $\mathrm{VO}_{2}$-Based MEMS Actuators. J. Microelectromech. S. 23, 243-251, doi:10.1109/JMEMS.2013.2271774 (2014).

\section{Acknowledgements}

This work was supported by a NSF award under the Grant No. DMR-1608899. H.S.C. acknowledges the Samsung Global Research Outreach program and K.D acknowledges the China Scholarship Council (CSC, No. 201406210211) for financial support. SEM and EDX performed in the Molecular Foundry were supported by the Office of Science, Office of Basic Energy Sciences, of the U.S. Department of Energy under Contract No. DEAC02-05CH11231. The authors thank Prof. Chris Dames for helpful discussion and Dr. D. Frank Ogletree, Dr. Shaul Aloni, and Mr. Ed Wong for technical assistance in in-situ SEM experiments.

\section{Author Contributions}

J.W. and H.S.C. designed the concept of the devices and experiments. H.S.C. fabricated the devices. H.S.C., J.S., C.K., S.L., J.P., Y.L., and K.W. performed the experiments. K.D. performed simulations. H.S.C., J.S., C.K., K.D., J.P., K.W., and J.W. analysed the data and wrote the paper. All authors discussed the results and commented on the manuscript.

\section{Additional Information}

Supplementary information accompanies this paper at doi:10.1038/s41598-017-07466-4

Competing Interests: The authors declare that they have no competing interests.

Publisher's note: Springer Nature remains neutral with regard to jurisdictional claims in published maps and institutional affiliations.

Open Access This article is licensed under a Creative Commons Attribution 4.0 International License, which permits use, sharing, adaptation, distribution and reproduction in any medium or format, as long as you give appropriate credit to the original author(s) and the source, provide a link to the Creative Commons license, and indicate if changes were made. The images or other third party material in this article are included in the article's Creative Commons license, unless indicated otherwise in a credit line to the material. If material is not included in the article's Creative Commons license and your intended use is not permitted by statutory regulation or exceeds the permitted use, you will need to obtain permission directly from the copyright holder. To view a copy of this license, visit http://creativecommons.org/licenses/by/4.0/.

(C) The Author(s) 2017 